\title{
Interplay between Kondo and Andreev-Josephson effects in a quantum dot coupled to one normal and two superconducting leads
}

\author{
Akira Oguri, ${ }^{1}$ Yoichi Tanaka ${ }^{2}$ and Johannes Bauer ${ }^{3,4}$ \\ ${ }^{1}$ Department of Physics, Osaka City University, Osaka 558-8585, Japan \\ ${ }^{2}$ Condensed Matter Theory Laboratory, RIKEN, Saitama 351-0198, Japan \\ ${ }^{3}$ Max-Planck Institute for Solid State Research, Heisenbergstr.1, 70569 Stuttgart, Germany \\ 4 Department of Physics, Harvard University, Cambridge, Massachusetts 02138, USA
}

(Dated: August 13, 2018)

\begin{abstract}
We study low-energy transport through a quantum dot coupled to one normal and two superconducting (SC) leads in a junction of Y-shape. In this geometry a crossover between Kondo dominated and Cooper-pairing dominated states occurs by tuning the parameters such as the quantized energy level $\epsilon_{d}$ of the dot and the Josephson phase $\phi$, which induces a supercurrent flowing between the two SC leads through the dot. Furthermore, Andreev scattering takes place at the interface between the dot and normal lead. The low-lying energy states of this system can be described by a local Fermi-liquid theory for interacting Bogoliubov particles. In a description based on an Anderson impurity model we calculate transport coefficients, renormalized parameters and spectral function, using Wilson's numerical renormalization group (NRG) approach, in the limit of large SC gap. Our results demonstrate how the Andreev resonance level approaches the Fermi level in the crossover region between Cooper-pairing singlet state and strong coupling situation as $\epsilon_{d}$ or $\phi$ are varied. The strong coupling situation shows a Kondo effect with a significantly renormalized resonance width. The crossover is smeared when the coupling between the dot and normal lead is large. Furthermore, asymmetry in the Josephson junction suppresses the cancellations of the SC proximity for finite $\phi$, and it favors the SC singlet state rather than the Kondo singlet.
\end{abstract}

PACS numbers: 73.63.Kv, 74.45.+c, 72.15.Qm

\section{INTRODUCTION}

The Kondo effect in superconducting (SC) materials has been one of the major topics in condensed matter physics over forty years. The energy gap $\Delta_{\mathrm{SC}}$ of a superconductor suppresses the magnetic screening of the conduction-electrons at low temperatures below the Kondo temperature $T_{K}$. The competition of these effects causes a quantum phase transition (QPT) between a magnetic-doublet and nonmagnetic-singlet ground states, which emerge at $\Delta_{\mathrm{SC}} \gg T_{K}$ and $\Delta_{\mathrm{SC}} \ll$ $T_{K}$, respectively $\underline{\underline{1}} \underline{\underline{-9}}$

The QPT has also been studied intensively for quantum dots $\stackrel{10}{=}$ and experiments have been carried out for carbon nanotube and semiconductor quantum dots $\underline{\underline{11}-15}$ One of the merits of quantum dots is the high tunability, and various types of the configurations can be examined. For instance, for a quantum dot (QD) embedded between two superconducting leads in a $\mathrm{SC} / \mathrm{QD} / \mathrm{SC}$ configuration, the competition between the Kondo and Josephson effects has also been expected to occur $\underline{\underline{16}} \underline{28}$ Furthermore, an interplay between Andreev scattering and the Kondo effect has been studied experimentally $\underline{29,30}$ and theoretically ${ }^{31-44}$ for a QD connected to a normal-metal (N) lead and a SC lead in a SC/QD/N configuration.

An interesting extension, which is also relevant experimentally ${ }_{25-47}$ is a junction of $\mathrm{Y}$-shape, at which a single QD is coupled to one normal and two superconducting (SC) leads as shown in Fig. 11 This system has been studied by Pala, Governale and König, using a real-time diagrammatic approach based on a pertur- bation expansion with respect to the tunneling matrix elements $\stackrel{48,49}{\underline{4}}$ Their calculations reveal precise features of the Josephson current and Andreev bound states both in equilibrium and nonequilibrium situations where a finite bias voltage is applied between the dot and the normal lead. However, their approach is not applicable at low temperatures $T<T_{K}$, and thus the competition between superconductivity and the Kondo effect occurring in this system has been left to be explored. Specifically, in the Y-junction the conduction electrons from the normal lead can screen the local moment induced in the QD. This changes the sharp transition between the magnetic and non-magnetic ground states into a crossover between

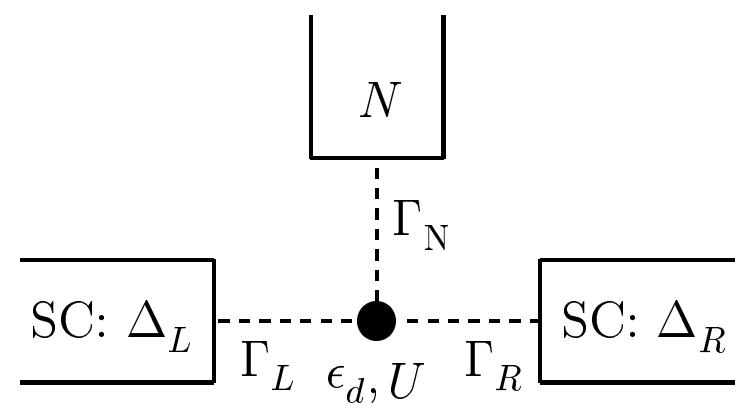

FIG. 1. Anderson impurity $(\bullet)$ coupled to one normal and two superconducting leads: $\epsilon_{d}$ and $U$ are the level position and Coulomb interaction. $\Gamma_{\nu} \equiv \pi \rho v_{\nu}^{2}$ with $\rho$ the density of states of the leads, and $v_{\nu}$ the tunneling matrix element $(\nu=L, R, N)$. The complex SC gap $\Delta_{L / R}=\left|\Delta_{L / R}\right| e^{i \theta_{L / R}}$ causes the Josephson current for finite $\phi \equiv \theta_{R}-\theta_{L}$. 
a Kondo singlet and a Cooper-pairing singlet. Furthermore, the Andreev scattering, which takes place between the QD and normal lead, can be controlled through the phase difference $\phi$ between the order parameters of two $\mathrm{SC}$ leads. This is because the SC proximity on the QD depends sensitively on the properties of the junctions, and thus on $\phi$. Conversely, the Josephson current flowing between the two SC leads is affected by the Andreev scattering of the conduction electrons from the normal lead.

The purpose of the present work is to study these interplays of the Kondo, Andreev, and Josephson effects which can be observed for the QD embedded in this three terminal system, $\stackrel{50}{=}$ To this end, we explore a wide region of the parameter space of this $\mathrm{Y}$-junction, varying the position of a quantized energy level $\epsilon_{d}$ of the QD modeled with an Anderson impurity, and also examine how an asymmetry of the Josephson junction affects the low-temperature properties. Specifically, we focus on the crossover between the ground states which can be classified into a Kondo singlet and a local Cooper-pairing singlet according to the fixed points of Wilson's numerical renormalization group (NRG) $\stackrel{51,52}{\underline{1}}$

For strongly correlated systems the Coulomb interaction $U$ is larger than the other energy scales, and for such cases the critical behavior near the QPT is scaled by a single parameter $\Delta_{\mathrm{SC}} / T_{K}$. In systems with QDs, however, some of the parameters can be tuned experimentally, and $U$ is not always the largest energy scale. Therefore, the ground-state properties depend on the other parameters, such as $\epsilon_{d}, U$, and the hybridizations between the dot and leads. Specifically, for small interactions $U<\Delta_{\mathrm{SC}}$, the SC pair correlations can penetrate into the QD and create a local Cooper pair, consisting of a linear combination of an empty state and a doubly occupied state. The essential physics of the local Cooper pairing can be deduced from the fixed point of the NRG in the limit of $\Delta_{\mathrm{SC}} \rightarrow \infty$, where the coherence becomes of the order of the lattice constant. We consider in detail this large SC gap limit in the present work.

The low-lying energy states of this Y-junction can be described by a local Fermi liquid of the interacting Bogoliubov particles. This is because the normal lead, coupled to the QD, has a continuous energy spectrum at the Fermi level. Furthermore, the local SC pair potential $\Delta_{d}$ is induced in the QD by the proximity effect. This $\Delta_{d}$ also plays a central role, and it varies sensitively with $\epsilon_{d}$ and $\phi$. For instance, the conductance due to Andreev scattering can be expressed at $T=0$ in terms of phase shift $\delta$ for the renormalized quasiparticles and the angle $\Theta_{B}$ of the Bogoliubov rotation determined by $\Delta_{d}$, and is enhanced at the crossover region between the Kondo singlet and local-Cooper pairing singlet $\underline{42-44}$ We also calculate the renormalized parameters for the interacting Bogoliubov particles. The results of the renormalization factor $z$, the Wilson ratio $R$, and the renormalized Andreev level $\widetilde{E}_{A}$ that corresponds to quasiparticle peak position appearing in the spectral function of the QD, provide us with sufficient information to understand the ground-state properties of the system thoroughly.

This paper is organized as follows. In Sec. III we introduce the single impurity Anderson model for the Yjunction, and provide some examples which capture typical behavior near the QPT occurring in a SC/QD/SC junction with a finite SC gap. In Sec. III, we give a local-Fermi liquid description for the interacting Bogoliubov particles in the large SC gap limit, and present the expressions of the correlation functions in terms of the renormalized parameters. Then in Sec. IV] we provide the NRG results for the spectral function, transport coefficient, and renormalized parameters. A summary is given in Sec. D

\section{MODEL AND FUNDAMENTAL ASPECTS}

\section{A. Model}

We start with the Anderson impurity model for a single quantum dot coupled to one normal $(N)$ and two superconducting (SC) leads,

$$
H=H_{d}+\sum_{\nu=N, L, R} H_{\nu}+\sum_{\nu=N, L, R} H_{T, \nu}+H_{\mathrm{SC}} .
$$

The explicit form of each part is given by,

$$
\begin{aligned}
H_{d}= & \xi_{d}\left(n_{d}-1\right)+\frac{U}{2}\left(n_{d}-1\right)^{2} \\
H_{\nu}= & \sum_{k, \sigma} \epsilon_{k} c_{\nu, k \sigma}^{\dagger} c_{\nu, k \sigma}, \\
H_{T, \nu}= & \sum_{\sigma} v_{\nu}\left(\psi_{\nu, \sigma}^{\dagger} d_{\sigma}+d_{\sigma}^{\dagger} \psi_{\nu, \sigma}\right), \\
H_{\mathrm{SC}}= & \sum_{k}\left(\Delta_{L} c_{L, k \uparrow}^{\dagger} c_{L,-k \downarrow}^{\dagger}+\text { H.c. }\right) \\
& +\sum_{k}\left(\Delta_{R} c_{R, k \uparrow}^{\dagger} c_{R,-k \downarrow}^{\dagger}+\text { H.c. }\right) .
\end{aligned}
$$

Here, $\xi_{d} \equiv \epsilon_{d}+U / 2$, and $U$ is the Coulomb interaction. The operator $d_{\sigma}^{\dagger}$ creates an electron with energy $\epsilon_{d}$ and spin $\sigma$ at the dot, and $n_{d}=\sum_{\sigma} d_{\sigma}^{\dagger} d_{\sigma}$. The operator $c_{\nu, k \sigma}^{\dagger}$ creates an electron with the energy $\epsilon_{k}$ in the leads $\nu(=N, L, R)$. The couplings between the dot and leads are described by the tunneling matrix elements $v_{\nu}$, and a linear combination of the conduction $\psi_{\nu, \sigma} \equiv \sum_{k} c_{\nu, k \sigma} / N_{\nu}$ with $N_{\nu}$ the number of the states in each lead. We assume that the density of states $\rho(\epsilon) \equiv \sum_{k} \delta\left(\epsilon-\epsilon_{k}\right) / N_{\nu}$ and $\Gamma_{\nu}(\epsilon) \equiv \pi v_{\nu}^{2} \rho(\epsilon)$ are constants independent of the frequency $\epsilon$ at $|\epsilon|<D$, where $D$ is the half band-width for the leads. The complex $s$ wave BCS gap, $\Delta_{L / R}=\left|\Delta_{L / R}\right| e^{i \theta_{L / R}}$ for the SC leads on the left $(L)$ and right $(R)$ induces a Josephson current when the phase difference $\phi \equiv \theta_{R}-\theta_{L}$ is finite. In this three terminal system, the current $J_{\nu}$ flowing from 


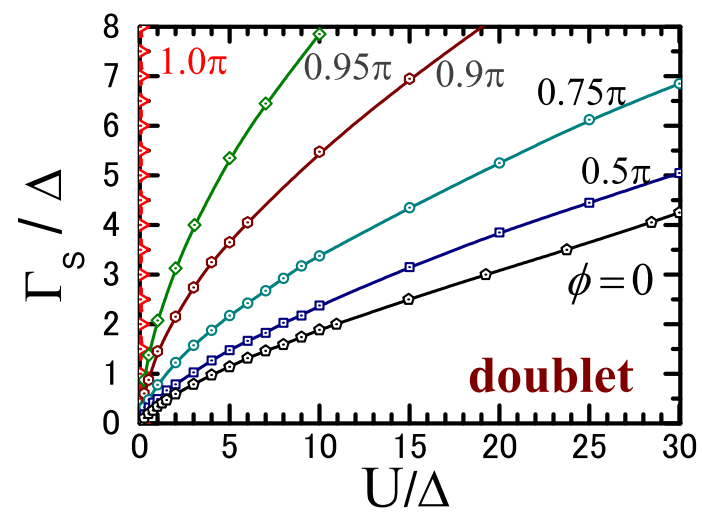

FIG. 2. (Color online) NRG results for the ground-state phase diagram of the Anderson impurity connected to two SC leads for several value of $\phi \equiv \theta_{R}-\theta_{L}$ in the electron-hole symmetric case $\epsilon_{d}=-U / 2$. In the upper and lower sides of each boundary the ground state is a nonmagnetic singlet and magnetic doublet, respectively. The Josephson junction is assumed to be symmetric $\Gamma_{L}=\Gamma_{R}\left(\equiv \Gamma_{S} / 2\right)$ and $\left|\Delta_{L}\right|=\left|\Delta_{R}\right|$ $(\equiv \Delta)$. The normal lead is not connected $\Gamma_{N}=0$ in this case.

the dot to the lead $\nu$ is given by

$$
J_{\nu}=\frac{i e}{\hbar} \sum_{\sigma} v_{\nu}\left(\psi_{\nu, \sigma}^{\dagger} d_{\sigma}-d_{\sigma}^{\dagger} \psi_{\nu, \sigma}\right) .
$$

Here, $-e$ denotes the electron charge with $e>0$.

The Hamiltonian $H$ contains a number of parameter regimes to be explored. We mainly consider the case where the couplings and the amplitude of the SC gaps are symmetric: $\Gamma_{L}=\Gamma_{R}\left(\equiv \Gamma_{S} / 2\right)$ and $\left|\Delta_{L}\right|=\left|\Delta_{R}\right|$ $(\equiv \Delta)$, for simplicity. The asymmetry in the Josephson coupling $\Gamma_{L} \neq \Gamma_{R}$ is also examined in the last part in Sec. IVC.

\section{B. QD connected two SC leads $\left(\Gamma_{N}=0\right)$}

Before discussing the three terminal case, we first of all consider a simpler case with $\Gamma_{N}=0$, where the normal lead is disconnected and the QD is coupled only to the two SC leads, in order to review some typical features of the competition between the Kondo and Josephson effects $\underline{8,22,23}, 25-27$ In this case the QPT occurs as a level crossing of the lowest two energy states of $H$, and thus the expectation value for the Josephson current and that for the order correlation functions show a discontinuous jump at the critical point.

In Fig. 2, the NRG results for the phase diagram of the ground state in the electron-hole symmetric case $\epsilon_{d}=-U / 2$ is plotted in a $U / \Delta$ vs $\Gamma_{S} / \Delta$ plane for several values of $\phi$. The upper (lower) side of each boundary correspond to the parameter region where the ground state is a non-magnetic singlet (magnetic doublet). These results clearly show that the magnetic-doublet region, appearing for large $U / \Delta$ or small $\Gamma_{S} / \Delta$, expands as $\phi$ increases. Therefore, the phase difference $\phi$ between the
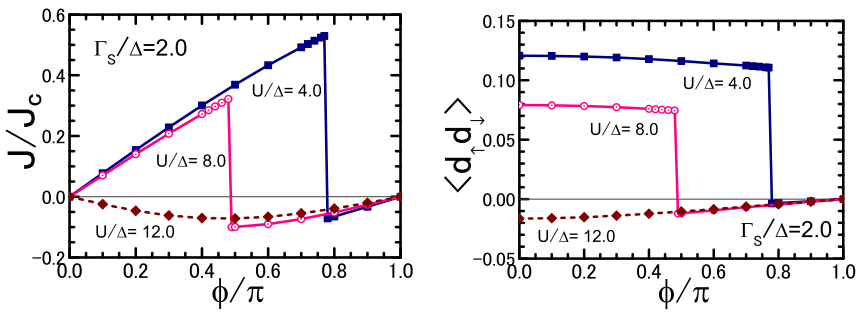

FIG. 3. (Color online) NRG results for the Josephson current and $\left\langle d_{\uparrow} d_{\downarrow}\right\rangle$ for the Anderson impurity connected to two SC leads are plotted as functions of $\phi$ for several value of $U$ in the electron-hole symmetric case $\epsilon_{d}=-U / 2$. The other parameters are chosen such that $\Gamma_{L}=\Gamma_{R}\left(\equiv \Gamma_{S} / 2\right),\left|\Delta_{L}\right|=\left|\Delta_{R}\right|$ $(\equiv \Delta), \Gamma_{S}=2.0 \Delta, \theta_{R}=-\theta_{L}(\equiv \phi / 2)$, and $\Gamma_{N}=0$. In this case the critical current is given by $J_{C}=e \Delta / \hbar$.

two SC order parameters tends to suppresses the Kondo screening, $\stackrel{22,23,25,26}{2}$ which in this case is carried out also by the quasiparticle excitations above the SC energy gap. As the value of $\phi$ increases from 0 to $\pi$, the SC proximity effect penetrating from the left lead and that from right lead cancel each other at the impurity site. This is because the SC proximity is determined by a superposition of $\Delta_{L}$ and $\Delta_{R}$, which can be explicitly seen in the impurity Green's function given in Eqs. (A2) and (A3). This suppression of the proximity effect leads to a reduction of the parameter region for singlet formation as seen in Fig. 2. Specifically, at $\phi=\pi$ the system is equivalent to an Anderson impurity model with an insulating bath,,$\underline{25,53}$ where for the particle-hole symmetric case and $U>0$ the ground state is always a doublet because the impurity level situates just on the Fermi level in the middle of the band gap.

Figure 3 shows the Josephson current $J$ and the SC correlation $\left\langle d_{\uparrow} d_{\downarrow}\right\rangle$ in the impurity site as functions of $\phi$ for several values of $U$. These ground-state averages vary discontinuously at the QPT, and take small negative values in the magnetic doublet ground state for finite SC gaps $\Delta$. These two expectation values in the doublet state are determined by the quasiparticle excitations above the SC energy gap $|\omega|>\Delta$, and vanish in the limit of $\Delta \rightarrow \infty$. The small negative values in the doublet state can be explained, for instance, using the perturbation expansion with respect to $1 / \Delta$ from the large gap limit $\underline{\underline{8}}$

\section{LARGE SC GAP LIMIT}

We consider the large gap limit, $\left|\Delta_{L / R}\right| \rightarrow \infty$, in the following since important features of the interplays between the Kondo effect and superconductivity in the Yjunction can be observed in this case although the quasiparticle excitations to the continuum-energy region above the SC gap have been projected out. For instance, the Andreev resonance state emerging inside the SC gap remains near the Fermi level, and thus the essential physics 
of the low-energy transport can be extracted from this case. Specifically, this limit describes reasonably the situation where the gap is much greater than the other energy scales, namely $\left|\Delta_{L / R}\right| \gg \max \left(\Gamma_{L}, \Gamma_{R}, \Gamma_{N}, U,\left|\epsilon_{d}\right|\right)$.

In the limit of $\left|\Delta_{L / R}\right| \rightarrow \infty$, the Hamiltonian $H$ can be mapped exactly onto a single-channel model, $, 10,42,54$

$$
\begin{aligned}
\mathcal{H}_{\mathrm{eff}} & =\mathcal{H}_{d S}+H_{d}+H_{N}+H_{T, N}, \\
\mathcal{H}_{d S} & =\Delta_{d} d_{\uparrow}^{\dagger} d_{\downarrow}^{\dagger}+\Delta_{d}^{*} d_{\downarrow} d_{\uparrow}, \\
\Delta_{d} & \equiv \Gamma_{R} e^{i \theta_{R}}+\Gamma_{L} e^{i \theta_{L}}=\left|\Delta_{d}\right| e^{i \theta_{d}} .
\end{aligned}
$$

Thus, the SC proximity effect becomes static in this case, and can be described by an additional term $\mathcal{H}_{d S}$ with the pair potential $\Delta_{d}$ penetrating into the QD. This term emerges as the Cooper pairs can be transferred between the dot and the SC leads even for large SC gaps whereas the unpaired quasiparticles cannot. The amplitude of $\Delta_{d}$ depends on the Josephson phase, and decreases as $\phi$ increases,

$$
\begin{aligned}
\left|\Delta_{d}\right| & =\Gamma_{S} \sqrt{1-\mathcal{T}_{0} \sin ^{2}(\phi / 2)}, \\
\Gamma_{S} & \equiv \Gamma_{R}+\Gamma_{L}, \quad \mathcal{T}_{0} \equiv \frac{4 \Gamma_{R} \Gamma_{L}}{\left(\Gamma_{R}+\Gamma_{L}\right)^{2}} .
\end{aligned}
$$

Specifically for the symmetric coupling $\Gamma_{R}=\Gamma_{L}$, the transmission probability in the normal-state case takes the value $\mathcal{T}_{0}=1$, and the amplitude is given simply by $\left|\Delta_{d}\right|=\Gamma_{S} \cos (\phi / 2)$ for $-\pi<\phi \leq \pi$.

\section{A. Bogoliubov particles}

The effective Hamiltonian $\mathcal{H}_{\text {eff }}$ can be transformed into an asymmetric Anderson model for the Bogoliubov particles, the total number of which is conserved ${ }^{25,42,43}$ In order to carry this out, we rewrite $\mathcal{H}_{\text {eff }}$ such that

$$
\begin{aligned}
\mathcal{H}_{\mathrm{eff}}= & {\left[d_{\uparrow}^{\dagger}, d_{\downarrow}\right]\left[\begin{array}{cc}
\xi_{d} & \Delta_{d} \\
\Delta_{d}^{*} & -\xi_{d}
\end{array}\right]\left[\begin{array}{l}
d_{\uparrow} \\
d_{\downarrow}^{\dagger}
\end{array}\right]+\frac{U}{2}\left(n_{d}-1\right)^{2} } \\
& +\sum_{j=-1}^{\infty} \sum_{\sigma} t_{j}\left(f_{j+1 \sigma}^{\dagger} f_{j \sigma}+f_{j \sigma}^{\dagger} f_{j+1 \sigma}\right)
\end{aligned}
$$

Here, the summation over $j$ describes the $H_{T, N}+H_{N}$ part with $f_{-1 \sigma} \equiv d_{\sigma}, f_{0 \sigma} \equiv \psi_{N, \sigma}$, and $t_{-1} \equiv v_{N}$. The operators $f_{j \sigma}$ for $j \geq 0$ correspond to a Wannier basis set for the conduction band. The explicit expression for $f_{j \sigma}$ and $t_{j}$ can be generated successively from the initial operator $f_{0 \sigma}$ and the energy spectrum $\epsilon_{k}$ of the conduction band, carrying out the Householder transformation 52 Therefore, no approximation has been made to obtain Eq. (9) from Eq. (4).

The effective Hamiltonian $\mathcal{H}_{\text {eff }}$ has a global U(1) symmetry in the Nambu pseudo-spin space along the direction $\boldsymbol{n} \propto\left(\left|\Delta_{d}\right| \cos \theta_{d},-\left|\Delta_{d}\right| \sin \theta_{d}, \xi_{d}\right)$. Thus, one can choose this direction $\boldsymbol{n}$ to be the quantization axis, carrying out a pseudo-spinor rotation,

$$
\left[\begin{array}{c}
\gamma_{j \uparrow} \\
(-1)^{j+1} \gamma_{j \downarrow}^{\dagger}
\end{array}\right]=\boldsymbol{U}^{\dagger}\left[\begin{array}{c}
f_{j \uparrow} \\
(-1)^{j+1} f_{j \downarrow}^{\dagger}
\end{array}\right],
$$

where

$$
\begin{aligned}
& \boldsymbol{U}=\left[\begin{array}{cc}
e^{i \frac{\theta_{d}}{2}} & 0 \\
0 & e^{-i \frac{\theta_{d}}{2}}
\end{array}\right]\left[\begin{array}{rr}
\cos \frac{\Theta_{B}}{2} & -\sin \frac{\Theta_{B}}{2} \\
\sin \frac{\Theta_{B}}{2} & \cos \frac{\Theta_{B}}{2}
\end{array}\right], \\
& \cos \frac{\Theta_{B}}{2}=\sqrt{\frac{1}{2}\left(1+\frac{\xi_{d}}{E_{A}}\right)}, \quad \cos \Theta_{B}=\frac{\xi_{d}}{E_{A}}, \\
& \sin \frac{\Theta_{B}}{2}=\sqrt{\frac{1}{2}\left(1-\frac{\xi_{d}}{E_{A}}\right)}, \quad \sin \Theta_{B}=\frac{\left|\Delta_{d}\right|}{E_{A}}, \\
& E_{A} \equiv \sqrt{\xi_{d}^{2}+\left|\Delta_{d}\right|^{2}} .
\end{aligned}
$$

Then, in terms of the Bogoliubov particles $\gamma_{j \sigma}$, the effective Hamiltonian takes the form

$$
\begin{aligned}
\mathcal{H}_{\mathrm{eff}}= & E_{A}\left(n_{\gamma,-1}-1\right)+\frac{U}{2}\left(n_{\gamma,-1}-1\right)^{2} \\
& +\sum_{j=-1}^{\infty} \sum_{\sigma} t_{j}\left(\gamma_{j+1 \sigma}^{\dagger} \gamma_{j \sigma}+\text { H.c. }\right) .
\end{aligned}
$$

Here, $n_{\gamma, j} \equiv \sum_{\sigma} \gamma_{j \sigma}^{\dagger} \gamma_{j \sigma}$, and $\epsilon_{d}^{\mathrm{eff}} \equiv E_{A}-U / 2$ corresponds to a bare impurity level for the Bogoliubov particles. This representation of the Hamiltonian clearly shows that the total number of Bogoliubov particles,

$$
\mathcal{N}_{\gamma} \equiv \sum_{j=-1}^{\infty} n_{\gamma, j}
$$

is conserved in the large gap limit as a result of the global U(1) symmetry. Furthermore, the Friedel sum rule holds

$$
\left\langle n_{\gamma,-1}\right\rangle=\frac{2}{\pi} \delta
$$

where $\delta$ is the phase shift of the Bogoliubov particles 55,56 Note that even in the case of $\xi_{d}=0$ where $\mathcal{H}_{\text {eff }}$ in the form of Eq. (9) has an electron-hole symmetry, the Bogoliubov particles do not have the particle-hole symmetry in the sense that $E_{A} \neq 0$, and thus $\left\langle n_{\gamma,-1}\right\rangle \neq 1$, as long as $\left|\Delta_{d}\right|$ is finite.

At low energies the interacting Bogoliubov particles, described by Eq. (15), show Fermi-liquid behavior that is characterized by the renormalized parameters:

$$
\begin{aligned}
\delta & \equiv \cot ^{-1}\left(\frac{\widetilde{E}_{A}}{\widetilde{\Gamma}_{N}}\right), \quad z^{-1}=1-\left.\frac{\partial \Sigma(\omega)}{\partial \omega}\right|_{\omega=0}, \\
\widetilde{\Gamma}_{N} & \equiv z \Gamma_{N}, \quad \widetilde{E}_{A} \equiv z\left[E_{A}+\Sigma(0)\right], \\
\widetilde{U} & \equiv z^{2} \Gamma_{\uparrow \downarrow ; \downarrow \uparrow}(0,0 ; 0,0) .
\end{aligned}
$$


Specifically, the Kondo energy scale can be deduced from the renormalization factor as $T_{K}=\pi \widetilde{\Gamma}_{N} / 4$. Furthermore, from the residual interaction $\widetilde{U}$ between the quasiparticles, the Wilson ratio $R$ can be deduced through

$$
R \equiv 1+\frac{\widetilde{U}}{\pi \widetilde{\Gamma}_{N}} \sin ^{2} \delta
$$

We calculate these renormalized parameters with the NRG through the convergence of the finite-size energy spectrum near the fixed point, $\underline{51,57}$ In Eq. (18)-(20),,$\Sigma(\omega)$ and $\Gamma_{\uparrow \downarrow ; \downarrow \uparrow}\left(\omega_{1}, \omega_{2} ; \omega_{3}, \omega_{4}\right)$ are the self-energy and vertex function, respectively, for the Bogoliubov particles, the retarded Green's function for which is defined by

$$
G_{\gamma}(\omega)=-i \int_{0}^{\infty} d t e^{i \omega t}\left\langle\left\{\gamma_{-1, \sigma}(t), \gamma_{-1, \sigma}^{\dagger}\right\}\right\rangle .
$$

Here, the spin suffix $\sigma$ is suppressed on the left-hand side because the Green's function for $\sigma=\uparrow$ and that for $\downarrow$ are identical due to the $\mathrm{SU}(2)$ symmetry for the real spin. The retarded Green's function for the electrons on the dot can be deduced from $G_{\gamma}(\omega)$ via the inverse Bogoliubov transform,

$$
\begin{aligned}
G_{d d}(\omega) & \equiv-i \int_{0}^{\infty} d t e^{i \omega t}\left\langle\left\{d_{\sigma}(t), d_{\sigma}^{\dagger}\right\}\right\rangle \\
& \Rightarrow G_{\gamma}(\omega) \cos ^{2} \frac{\Theta_{B}}{2}-\left\{G_{\gamma}(-\omega)\right\}^{*} \sin ^{2} \frac{\Theta_{B}}{2} .
\end{aligned}
$$

\section{B. Conductance and Current}

The low-energy transport, deduced from Eq. (15), can also be described by the local Fermi liquid theory. At $T=0$, the occupation number of the electrons $\left\langle n_{d}\right\rangle$ and the SC pair correlation $\left\langle d_{\downarrow} d_{\uparrow}\right\rangle$ in the QD are determined by the occupation of the Bogoliubov particles $\left\langle n_{\gamma,-1}\right\rangle=$ $2 \delta / \pi$ defined in Eq. (16), and the Bogoliubov angle $\Theta_{B}$,

$$
\begin{aligned}
\left\langle n_{d}\right\rangle-1 & =\left(\left\langle n_{\gamma,-1}\right\rangle-1\right) \cos \Theta_{B}, \\
\left\langle d_{\downarrow} d_{\uparrow}\right\rangle & =\frac{1}{2}\left(\left\langle n_{\gamma,-1}\right\rangle-1\right) e^{i \theta_{d}} \sin \Theta_{B} .
\end{aligned}
$$

Note that $\cos \Theta_{B}=\xi_{d} / E_{A}, e^{i \theta_{d}} \sin \Theta_{B}=\Delta_{d} / E_{A}$, and the phase of $\left\langle d_{\downarrow} d_{\uparrow}\right\rangle$ is given by $\theta_{d}$, which coincides with the phase of the local gap $\Delta_{d}$. The occupation number of the electron $\left\langle n_{d}\right\rangle-1$ and $\left\langle d_{\downarrow} d_{\uparrow}\right\rangle$ correspond to the $z$ component and the projection on the $x-y$ plane of the local pseudo-spin moment induced on the impurity site ${ }^{25}$ Specifically, in the electron-hole symmetric case $\xi_{d}=0$, the local level is given by $E_{A}=\left|\Delta_{d}\right|$ and thus the Bogoliubov angle is locked at $\Theta_{B}=\pi / 2$.

The dc conductance $g_{N S}$ due to the Andreev scattering between the dot and the normal lead can also be expressed, at $T=0$, in terms of the phase shift $\delta$ and

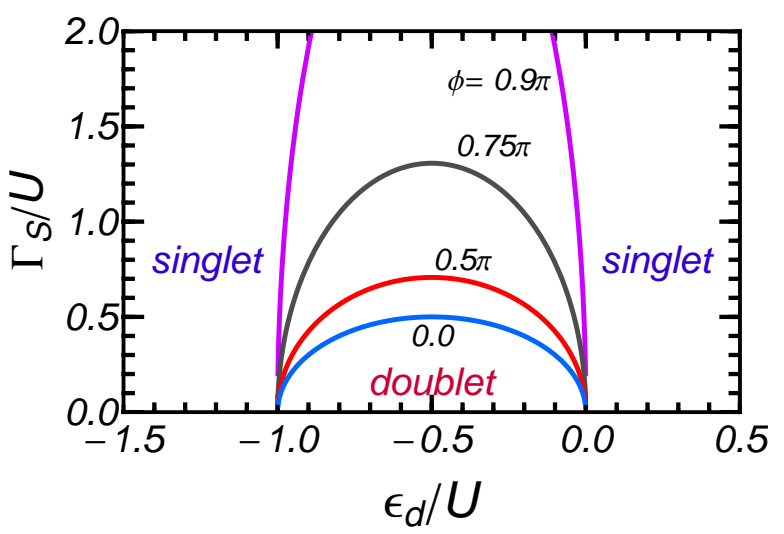

FIG. 4. (Color online) Ground-state phase diagram in the limit of $\Gamma_{N} \rightarrow 0$ and $\left|\Delta_{L / R}\right| \rightarrow \infty$ is plotted in $\epsilon_{d}$ vs $\Gamma_{S}$ plane for several values of $\phi$. The couplings between the QD and SC leads is chosen to be symmetric $\Gamma_{L}=\Gamma_{R}\left(\equiv \Gamma_{S} / 2\right)$. In this case the local SC gap is given by $\Delta_{d}=\Gamma_{S} \cos \phi / 2$ for $|\phi| \leq \pi$. The ground state is a singlet due to the local Cooper pairing outside the semi ellipse of $\sqrt{\left(\epsilon_{d}+U / 2\right)^{2}+\left|\Delta_{d}\right|^{2}}=U / 2$. Inside this semi ellipse the ground state is a magnetic doublet, which for finite $\Gamma_{N}$ changes to a Kondo singlet due to the conduction-electron screening of the local moment. Note that at $\epsilon_{d}=-0.5 U$ the system has the electron-hole symmetry, and the results for this case is given in Fig. 2 at finite SC gaps.

Bogoliubov angle $\Theta_{B} \stackrel{42}{\stackrel{42}{2}}$

$$
\begin{aligned}
g_{N S} & =4 \Gamma_{N}^{2}\left|\left\{\boldsymbol{G}_{d d}^{r}(\omega=0)\right\}_{12}\right|^{2} \\
& =\frac{4 e^{2}}{h} \sin ^{2} \Theta_{B} \sin ^{2} 2 \delta .
\end{aligned}
$$

Here, $\left\{\boldsymbol{G}_{d d}^{r}(\omega)\right\}_{12}=\left\langle\left\langle d_{\uparrow} ; d_{\downarrow}\right\rangle_{\omega}\right.$ is the off-diagonal (anomalous) component of the retarded Green's function in the Nambu pseudo-spin formalism, the corresponding Matsubara function of which is defined in Appendix A. Therefore, the zero-temperature conductance $g_{N S}$ is determined by the value at the Fermi level $\omega=0$. The argument $2 \delta$ in Eq. (26) appears as a difference between the phase shift for the quasi-particle $+\delta$ and that for the quasi-hole $-\delta$.

In the large gap limit, the Josephson current flowing through the dot can also be expressed in terms of the Bogoliubov angle $\Theta_{B}$ and the phase shift $\delta$, or $\left\langle n_{\gamma,-1}\right\rangle$ [see Appendix $\mathrm{A}$,

$$
\langle J\rangle=J_{C} \mathcal{T}_{0} \frac{\left|\left\langle n_{\gamma,-1}\right\rangle-1\right| \sin \Theta_{B} \sin \phi}{2 \sqrt{1-\mathcal{T}_{0} \sin ^{2}(\phi / 2)}} .
$$

Here, $J_{C} \equiv e \Gamma_{S} / \hbar$ is the critical current. Note that $\langle J\rangle$ shows a non-sinusoidal dependence on $\phi$ in general because $\Theta_{B},\left\langle n_{\gamma,-1}\right\rangle$, and the denominator of Eq. (27) that arises through $\left|\Delta_{d}\right|$ vary as functions of $\phi$. 


\section{Kondo singlet vs Local Cooper pairing}

The ground state of the asymmetric Anderson model, given in Eq. (15), can be classified according to the fixed points of the NRG 51,52 Among them the strong-coupling fixed point describes the Kondo singlet, for which the impurity site is singly occupied. The frozen-impurity fixed point describes a different situation, where the impurity level is far away from the Fermi level and the impurity site becomes empty or doubly occupied. In our case, the frozen-impurity fixed point is defined with respect to Eq. (15) for the Bogoliubov particles, and thus this describes a singlet state by a local Cooper pairing that consists of a linear combination of the empty and doubly occupied impurity states of the electrons represented in Eq. (9). We refer to this fixed point as local Cooper pairing in the following.

The ground state of $\mathcal{H}_{\text {eff }}$ varies continuously between the Kondo singlet and the local-Cooper pairing, depending on the Hamiltonian parameters $E_{A}, U$, and $\Gamma_{N}$. A rough sketch of the ground-state phase diagram can be obtained quickly from that in the atomic limit $\Gamma_{N} \rightarrow 0$, where the normal lead is disconnected. In this limit the dot is occupied by a single Bogoliubov particle with spin $1 / 2$ for $E_{A}<U / 2$ whereas the dot is empty with no Bogoliubov particle for $E_{A}>U / 2$. Thus, the phase boundary is given by a simple semi ellipse of $\sqrt{\xi_{d}^{2}+\left|\Delta_{d}\right|^{2}}=$

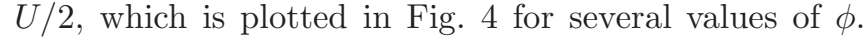
The ground state is a singlet due to the local Cooper pairing outside the semi ellipse whereas inside the semielliptic boundary the ground state is a doublet and thus the local moment arises in this limit of $\Gamma_{N} \rightarrow 0$. However, the local moment is screened when the normal lead is connected $\Gamma_{N} \neq 0$, and then the ground state inside the semi ellipse changes to the Kondo singlet. The coupling to the normal lead also changes the sharp transition at the boundary to a continuous crossover between the local-Cooper-pairing singlet and the Kondo singlet. $\underline{42}$

There are further quantitative corrections when the SC gap $\Delta$ is finite. This was also examined for the $\Gamma_{N}=$ 0 case, using the NRG $\underline{6}, \frac{7}{7}$ The results showed that the region of the magnetic doublet state becomes small as $\Delta$ decreases. This is because also the excited states in the SC leads above the gap $|\omega|>\Delta$ contribute to the screening of the local moment for finite $\Delta$.

\section{NRG RESULTS}

In this section, we provide the NRG results for the ground-state properties of the $\mathrm{Y}$-junction in the large gap limit $\left|\Delta_{L / R}\right| \rightarrow \infty$.

\section{A. Spectral function}

We first of all discuss the impurity spectral function for electrons $A_{d d}=(-1 / \pi) \operatorname{Im} G_{d d}$ that can be deduced from the one for the Bogoliubov particles $A_{\gamma}=(-1 / \pi) \operatorname{Im} G_{\gamma}$,

$$
A_{d d}(\omega)=A_{\gamma}(\omega) \cos ^{2} \frac{\Theta_{B}}{2}+A_{\gamma}(-\omega) \sin ^{2} \frac{\Theta_{B}}{2} .
$$

Specifically, the spectrum is symmetric $A_{d d}(-\omega)=$ $A_{d d}(\omega)$ in the electron-hole symmetric case, where $\Theta_{B}=$ $\pi / 2$. Note that the single-electron spectrum $A_{d d}(\omega)$ consists of a superposition of a single-Bogoliubov-particle part $A_{\gamma}(\omega)$ and a single-Bogoliubov-hole part $A_{\gamma}(-\omega)$. This can be deduced from the Lehmann representation that is expressed in terms of the matrix element $\left\langle m, N_{b}^{\prime}\left|d_{\uparrow}^{\dagger}\right| \mathrm{GS}, N_{b}\right\rangle$ between the ground state $\left|\mathrm{GS}, N_{b}\right\rangle$ and an excited state $\left|m, N_{b}^{\prime}\right\rangle$ of $\mathcal{H}_{\text {eff }}$, where $N_{b}$ is an eigenvalue of the total number of the Bogoliubov particles $\mathcal{N}_{\gamma}$ defined in Eq. (16). This matrix element can be finite not only for the single-particle excitations with $N_{b}^{\prime}=N_{b}+1$ but also single-hole excitations with $N_{b}^{\prime}=N_{b}-1$ of the Bogoliubov particles because the electron $d_{\uparrow}^{\dagger}$ can be decomposed into a superposition of the annihilation $\gamma_{-1, \uparrow}^{\dagger}$ and creation $\gamma_{-1, \downarrow}$ of the Bogoliubov particles.

The low-energy spectral weight is dominated by the renormalized Andreev resonances that appear in $A_{d d}(\omega)$ as a pair of quasiparticle peaks,

$$
A_{d d}(\omega) \simeq \frac{z}{\pi}\left[\frac{\widetilde{\Gamma}_{N} \cos ^{2} \frac{\Theta_{B}}{2}}{\left(\omega-\widetilde{E}_{A}\right)^{2}+\widetilde{\Gamma}_{N}^{2}}+\frac{\widetilde{\Gamma}_{N} \sin ^{2} \frac{\Theta_{B}}{2}}{\left(\omega+\widetilde{E}_{A}\right)^{2}+\widetilde{\Gamma}_{N}^{2}}\right] .
$$

The peak position $\pm \widetilde{E}_{A}$, width $\widetilde{\Gamma}_{N}$, and renormalization factor $z$ vary as the Coulomb repulsion increases from $U=0$, for which we have $\widetilde{E}_{A}=E_{A}, \widetilde{\Gamma}_{N}=\Gamma_{N}$, and $z=1$.

The high-energy part of the spectral weight away from the Fermi level can be inferred from the one in the atomic limit $\Gamma_{N}=0$. For weak repulsions $E_{A}>U / 2$, the ground state is a singlet, and $A_{d d}(\omega)$ has two peaks at $\omega= \pm\left(E_{A}-U / 2\right)$. This is because in this case $A_{\gamma}(\omega)$ has a single peak at $\omega=E_{A}-U / 2$, which moves towards the Fermi level as $U$ increases. On the other hand, for strong repulsions $E_{A}<U / 2$ in the $\Gamma_{N} \rightarrow 0$ limit, the ground state is a magnetic doublet, and then $A_{d d}(\omega)$ has four peaks emerging at $\omega= \pm \epsilon_{\mathrm{UP}}$ and $\pm \epsilon_{\mathrm{LW}}$. These peaks are caused by the excitations to the upper $\epsilon_{\mathrm{UP}} \equiv U / 2+E_{A}$ and lower $\epsilon_{\mathrm{LW}} \equiv-\left(U / 2-E_{A}\right)$ atomic peaks defined with respect to the Bogoliubov particles, and each of the two final states consists of a linear combination of an empty and doubly occupied states of the original electrons. These peaks correspond to the original Andreev bound states typically found in situations of a QD coupled only to a superconductor, and they are deltafunctions in the limit $\Gamma_{N}=0$. For finite superconducting gap their position and the occurrence of the ground state transition change quantitatively $\underline{\underline{7}}$ In this situation in the doublet phase it is possible that the higher excitations $\pm \epsilon_{\mathrm{UP}}$ are not found within the gap anymore. 

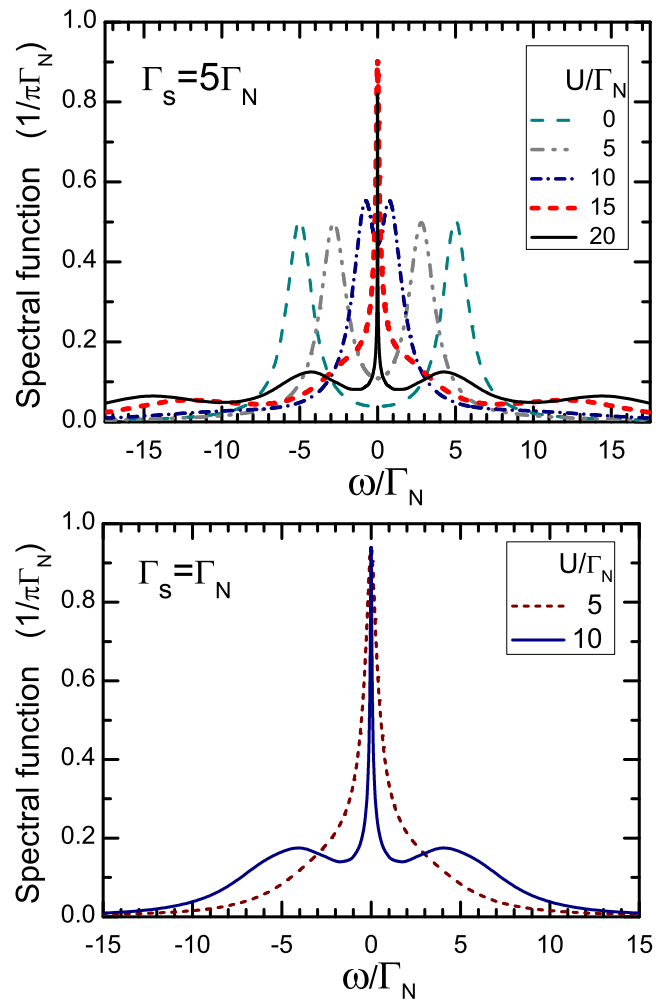

FIG. 5. (Color online) NRG results for the Spectral function $A_{d d}(\omega)=(-1 / \pi) \operatorname{Im} G_{d d, \sigma}(\omega)$, in the large gap limit $\left|\Delta_{L / R}\right| \rightarrow \infty$ at $\phi=0$, is plotted for several value of $U$ choosing the couplings to the SC leads such that (upper panel) $\Gamma_{S} / \Gamma_{N}=5.0$, and (lower panel) $\Gamma_{S} / \Gamma_{N}=1.0$. The other parameters are taken to be $\Gamma_{L}=\Gamma_{R}\left(\equiv \Gamma_{S} / 2\right)$ assuming the electron-hole symmetric $\epsilon_{d}=-U / 2$. In the present case the local SC gap in the impurity site is given by $\Delta_{d}=\Gamma_{S}$, and $E_{A}=\Gamma_{S}$.

Figure 5 shows the spectral function for $\phi=0$ in the electron-hole symmetric case, where $E_{A}=\Gamma_{S}$. In the upper panel $A_{d d}(\omega)$ in the case of relatively small $\Gamma_{N}$ with $E_{A}=5 \Gamma_{N}$ is plotted for several values of the Coulomb repulsion $U / \Gamma_{N}=0,5,10,15,20$. In these examples, at $U=10 \Gamma_{N}$ the bare parameter takes the value $E_{A}=U / 2$, and thus the dash-dot line represents the results obtained at the crossover region between the Kondo and local SC singlet states. The pair of renormalized Andreev resonances at $\omega \simeq \pm \widetilde{E}_{A}$, which correspond to the Kondo peak for the Bogoliubov particles described in Eq. (29), shift closer to the Fermi level $\omega=0$ as the Coulomb repulsion $U$ increases from 0 to $2 E_{A}$. Then, for $U>2 E_{A}$ five peaks emerge as seen the curves for $U=15 \Gamma_{N}$ and $20 \Gamma_{N}$. Among them, the central peak near the Fermi level $\omega=0$ corresponds to the Kondo resonance for the Bogoliubov particles, which appears for $A_{\gamma}(\omega)$ at small positive frequency $\omega \simeq \widetilde{E}_{A}$. However, for the electron spectral function $A_{d d}(\omega)$, this peak at $\omega \simeq \widetilde{E}_{A}$ and the counterpart for holes at $\omega \simeq-\widetilde{E}_{A}$ overlap to form a single peak at the Fermi energy $\omega=0$. The other four peaks represent the electron and hole components of the excitations to the the upper and lower atomic levels of the Bogoliubov particles. For instance, in the curve for $U=20 \Gamma_{N}$, the broad peak at $\omega \simeq-5 \Gamma_{N}$ and the one at $\omega \simeq+15 \Gamma_{N}$ correspond to upper and lower atomic peaks appearing in $A_{\gamma}(\omega)$, respectively.

The lower panel of Fig. 5 shows the spectral function, obtained at $E_{A}=\Gamma_{N}$ where the bare Andreev level and the hybridization energy due to the coupling to the normal lead coincide. The Coulomb interaction is chosen to be $U=5 \Gamma_{N}$ and $10 \Gamma_{N}$, so that $E_{A}<U / 2$ for these two cases. As the coupling to the normal lead $\Gamma_{N}$ is relatively large in these cases the sub peaks of the Coulomb oscillation are smeared especially in the curve for $U=5 \Gamma_{N}$. Nevertheless, in the other curve for $U=10 \Gamma_{N}$, the sharp central peak and two sub peaks of the atomic nature are distinguishable. The central peak emerges as a results of the superposition of the two renormalized Andreev level at $\omega \simeq \pm \widetilde{E}_{A}$ close to the Fermi level whereas each of the sub peaks at $\omega \simeq \pm 4.0 \Gamma_{N}$ emerge as a sum of the sub peaks in $A_{\gamma}(\omega)$ and that in the counterpart for the holes $A_{\gamma}(-\omega)$.

The results which we have presented in Fig. 5 have been the ones obtained at $\phi=0$, where there is no phase difference between the two SC order parameters. As we have chosen the parameters such that $\xi_{d}=0$ and $\Gamma_{L}=$ $\Gamma_{R}$, the dependence of $A_{d d}(\omega)$ on $\phi$ enters only through the bare Andreev level $E_{A}=\Gamma_{S} \cos \phi / 2$ in this case. Therefore, the results capture essential features common to the case for finite $\phi$. In the electron-hole asymmetry case $\xi_{d} \neq 0$, however, $A_{d d}(\omega)$ is no longer a symmetric function of $\omega$. The asymmetry in the $\omega$ dependence enters through $\Theta_{B}$ as it deviates from $\pi / 2$ for $\xi_{d} \neq 0$.

\section{B. Transport properties \& Fermi-liquid behavior}

In this subsection we present the NRG results for the ground-state properties of the Y-junction for symmetric coupling $\Gamma_{L}=\Gamma_{R}\left(=\Gamma_{S} / 2\right)$, where $\mathcal{T}_{0}=1$. The Josephson phase is chosen such that $\theta_{R}=-\theta_{L}(=\phi / 2)$, which makes the local SC gap $\Delta_{d}$ and pair correlation $\left\langle d_{\downarrow} d_{\uparrow}\right\rangle$ real $\theta_{d}=0$ as shown in Appendix. We consider the electron-hole symmetric case $\xi_{d}=0$ in Sec. IVB 1 and then discuss the gate voltage dependence varying $\xi_{d}$ in Sec. IVB2,

\section{Electron-hole symmetric case}

Figure 6 shows the results of the phase shift $\delta$, the SC pair correlation $2\left|\left\langle d_{\downarrow} d_{\uparrow}\right\rangle\right|$, the conductance $g_{N S}$, and the Josephson current $J$ as functions of $\phi$ for several values of $\Gamma_{N} / \Gamma_{S}$ in the electron-hole symmetric case, where the Bogoliubov angle is locked at $\Theta_{B}=\pi / 2$ and $\left\langle n_{d}\right\rangle=1$, as mentioned. Thus, the ground state properties are determined by the phase shift $\delta$, which depends on the Josephson phase through $E_{A}=\Gamma_{S} \cos (\phi / 2)$. The Coulomb 

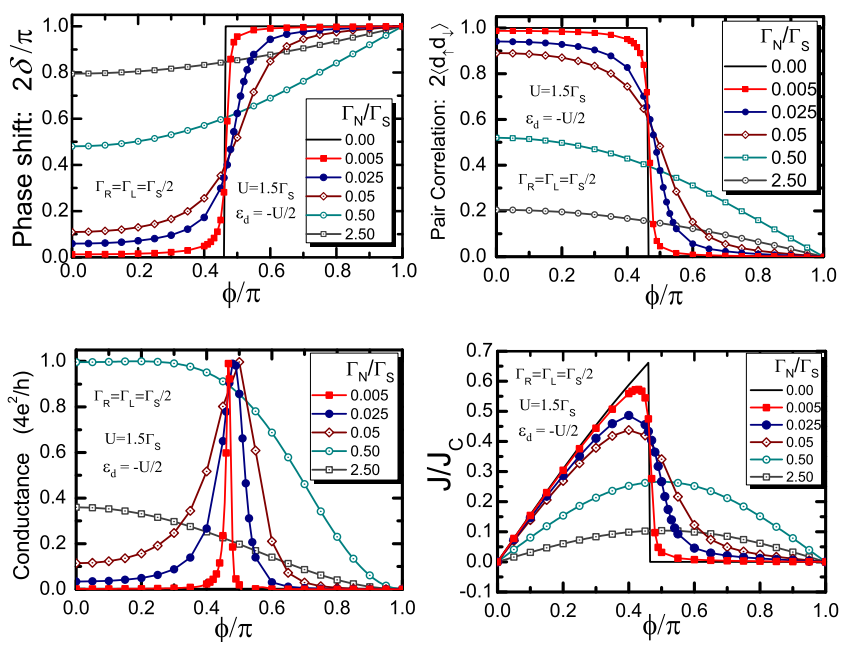

FIG. 6. (Color online) Phase shift and some related groundstate averages are plotted vs Josephson phase $\phi$ for several values of $\Gamma_{N}$ in the electron-hole symmetric case $\epsilon_{d}=-U / 2$ : (upper panel) phase shift $\delta$ and pair correlation $2\left|\left\langle d_{\downarrow} d_{\uparrow}\right\rangle\right|$, (lower panel) conductance $g_{N S}$ and Josephson current $J$ in units of $J_{C}=e \Gamma_{S} / \hbar$. The other parameters are chosen such that $\Gamma_{L}=\Gamma_{R}\left(=\Gamma_{S} / 2\right)$, and $U=1.5 \Gamma_{S}$ in the large gap limit $\left|\Delta_{L / R}\right| \rightarrow \infty$.

repulsion is chosen to be $U=1.5 \Gamma_{S}$, and for this interaction the QPT occurs at $\phi \simeq 0.46 \pi$ in the $\Gamma_{N} \rightarrow 0$ limit.

The coupling to the normal lead makes the excitation spectrum of the dot gapless and changes the sharp QPT into a continuous crossover between the two different singlet states, namely the Kondo and local SC singlets. For $\phi \lesssim 0.46 \pi$, the ground state is the local Cooper pairing consisting of a linear combination of the empty and doubly occupied impurity states with small $\delta$. For larger phase difference, $\phi \gtrsim 0.46 \pi$, however, the ground state is a Kondo singlet state, for which $\delta \simeq \pi / 2$.

The phase shift shown in the upper left panel of Fig. 6] can be expressed as the number of Bogoliubov particles on the impurity site, $\left\langle n_{\gamma,-1}\right\rangle=2 \delta / \pi$, due to the Friedel sum rule given in Eq. (17). Furthermore in the electron-hole symmetric case, the SC pair correlation defined in Eq. (25) is given simply by $2\left\langle d_{\uparrow} d_{\downarrow}\right\rangle=1-\left\langle n_{\gamma,-1}\right\rangle$. Therefore, the pair correlation is suppressed in the Kondo regime for $\phi \gtrsim 0.46 \pi$, as seen in the upper right panel.

The Andreev conductance $g_{N S}$, shown in the lower left panel of Fig. 6, also varies as a function of the Josephson phase $\phi$. At the crossover region between the Kondo singlet and local-Cooper-pairing states, the conductance due to the Andreev scattering $g_{N S}$ has a sharp peak for small $\Gamma_{N}$. The crossover behavior, however, is smeared as $\Gamma_{N}$ increases. The conductance takes the unitary limit value $4 e^{2} / h$ at $\delta=\pi / 4$ where the renormalized resonance width and the renormalized Andreev level coincide such that $\widetilde{\Gamma}_{N}=\widetilde{E}_{A}$. This happens in Fig. [6] at $\phi \simeq 0.0$ for $\Gamma_{N}=0.5 \Gamma_{S}$.

The Josephson current, in the lower right panel, also
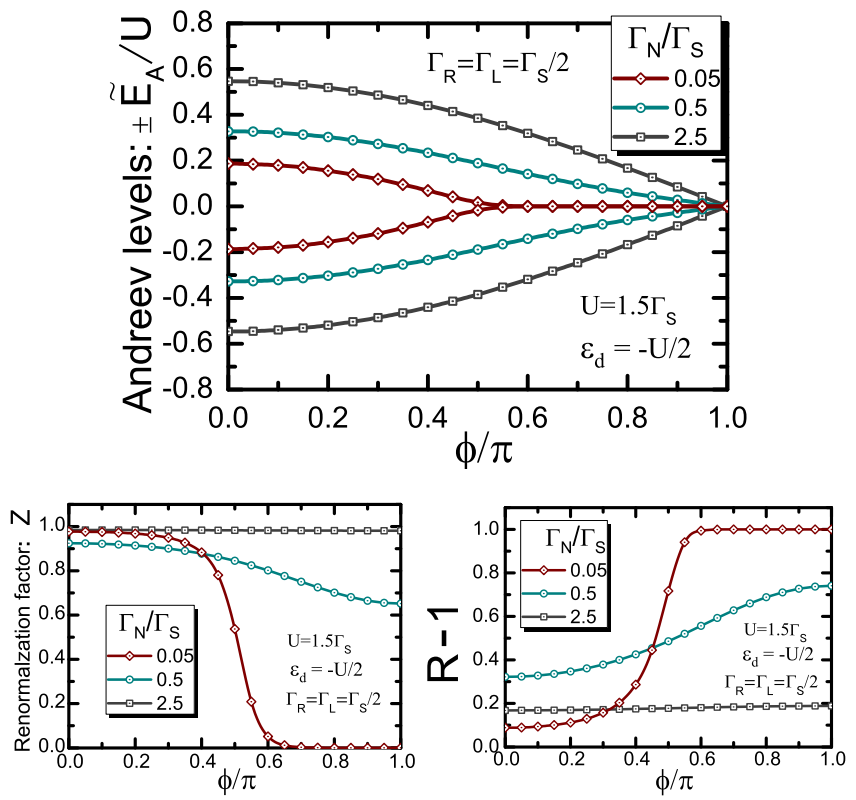

FIG. 7. (Color online) Renormalized parameters are plotted vs Josephson phase $\phi$ for several values of $\Gamma_{N}$ in the electronhole symmetric case $\epsilon_{d}=-U / 2$ : (upper panel) renormalized Andreev level $\pm \widetilde{E}_{A}$, (lower panel) renormalization factor $z$ and Wilson ratio $R$. The other parameters are chosen such that $\Gamma_{L}=\Gamma_{R}\left(=\Gamma_{S} / 2\right)$, and $U=1.5 \Gamma_{S}$ in the large gap limit $\left|\Delta_{L / R}\right| \rightarrow \infty$.

shows the crossover behavior near $\phi \simeq 0.46 \pi$, and decreases at $\phi \gtrsim 0.46 \pi$ when $\Gamma_{N}$ is small. The value of the current approaches zero in the Kondo-singlet region since in this case the large gap limit has been taken. For finite SC gaps, however, a weak current will flow in the opposite direction as seen in Fig. 3 for the magnetic-doublet state. Similarly, for finite SC gaps, the SC pair correlation $\left\langle d_{\uparrow} d_{\downarrow}\right\rangle$ will also change the sign at crossover, and has a small negative value in the magnetic ground state.

We have also deduced the renormalized parameters for the local Fermi liquid of the interacting Bogoliubov particles from the convergence of the finite-size energy spectrum in the successive NRG steps 51,57 Figure 7 shows the results for the renormalized Andreev level $\pm \widetilde{E}_{A}$, the wavefunction renormalization factor $z$, and the Wilson ratio $R$. We see for small $\Gamma_{N}\left(=0.05 \Gamma_{S}\right)$ that the parameters are significantly renormalized in the Kondo singlet state for $\phi \gtrsim 0.46 \pi$, where $z \ll 1.0, R \simeq 2.0$, and the pair of renormalized Andreev peaks $\pm \widetilde{E}_{A}$ lie close to the Fermi level $\omega=0$. This indicates that the Bogoliubov particles are strongly correlated in the Kondo regime. In contrast, in the local Cooper-pairing state for $\phi \lesssim 0.46 \pi$, the parameters are not renormalized so much $z \simeq 1.0$, $R \simeq 1.1$, and the renormalized Andreev peaks $\pm \widetilde{E}_{A}$ situate away from the Fermi level. As the coupling $\Gamma_{N}$ between the QD and normal lead becomes large, these two singlet states become indistinguishable, as those for $\Gamma_{N}=2.5 \Gamma_{S}$ seen in Fig. 7 

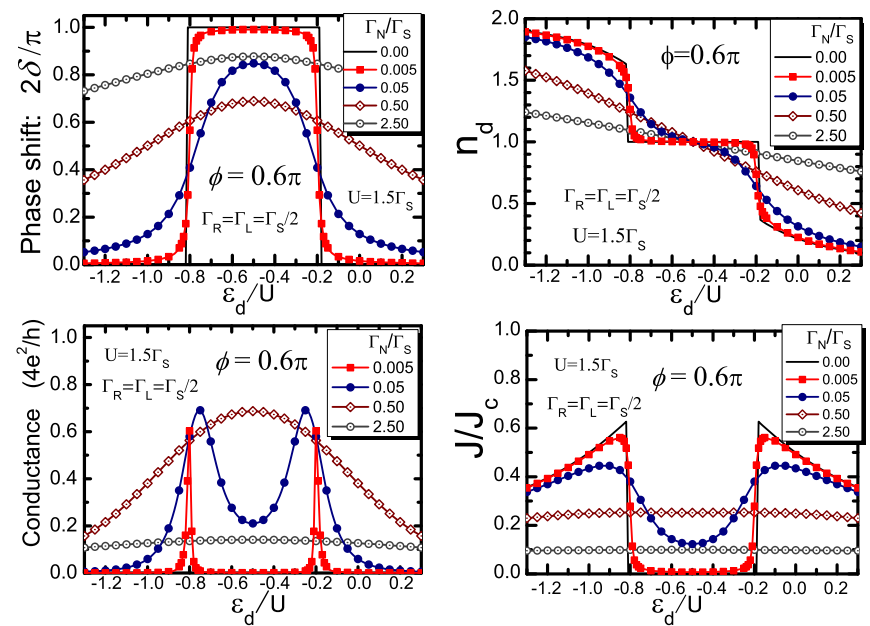

FIG. 8. (Color online) Phase shift and some related groundstate averages are plotted vs $\epsilon_{d} / U$ for $\phi=0.6 \pi$ for several values of $\Gamma_{N}$ : (upper panel) phase shift $\delta$ and electron occupation number $\left\langle n_{d}\right\rangle$, (lower panel) conductance $g_{N S}$ and Josephson current $J$ in units of $J_{C}=e \Gamma_{S} / \hbar$. The parameters are chosen to be $\Gamma_{L}=\Gamma_{R}\left(=\Gamma_{S} / 2\right)$, and $U=1.5 \Gamma_{S}$.

\section{Electron-hole asymmetric case}

We next consider the electron-hole asymmetric case, in which the Bogoliubov angle $\Theta_{B}=\cot ^{-1}\left(\xi_{d} /\left|\Delta_{d}\right|\right)$ deviates from $\pi / 2$ as $\xi_{d}$ varies. Furthermore, the phase shift $\delta$ also varies as a a function of $\xi_{d}$ since the bare position $E_{A}-U / 2$ of the Andreev level depends on $\xi_{d}$. Through these changes of the phase parameters $\Theta_{B}$ and $\delta$, the ground-state properties of this $\mathrm{Y}$-junction depend on the gate voltage $\epsilon_{d}$.

In this subsection we examine the $\epsilon_{d}$ dependence for several values of the Josephson phase $\phi=0.3 \pi, 0.46 \pi$, and $0.6 \pi$, choosing the Coulomb repulsion to be $U=$ $1.5 \Gamma_{S}$ as that in the half-filled case discussed in the above. The phase boundary between the singlet and doublet ground states moves in the $\Gamma_{S}$ vs $\epsilon_{d}$ plane as $\phi$ increases as shown in Fig. 4. In our parameter set $\Gamma_{S} / U=0.666$, and the QPT occurs for $\phi=0.6 \pi$ when $\epsilon_{d}$ varies in the range $-1.0 \leq \epsilon_{d} / U \leq 0.0$, whereas the level crossing does not occur for $\phi=0.3 \pi$. A marginal situation is realized for $\phi \simeq 0.46 \pi$, in this case the system approaches very closely to the phase boundary near the symmetric point $\epsilon_{d} \simeq-U / 2$.

In Fig. 8, the correlation functions for $\phi=0.6 \pi$ are plotted vs $\epsilon_{d} / U$. The ground state changes discontinuously in the limit of $\Gamma_{N}=0$, at $\epsilon_{d} \simeq-0.82 U$ and $-0.18 U$. The sharp transition becomes a continuous crossover for finite $\Gamma_{N}$, and at $-0.82 U \lesssim \epsilon_{d} \lesssim-0.18 U$ the ground state is a Kondo singlet consisting of the strong correlated Bogoliubov particles, and the electron filling is almost $\left\langle n_{d}\right\rangle \simeq 1.0$, as shown in the upper right panel. In the upper left panel, we can see that in this region of $\epsilon_{d}$ the occupation number of the Bogoliubov particles at the impurity level, $\left\langle n_{\gamma,-1}\right\rangle=2 \delta / \pi$, decreases as $\Gamma_{N}$ in-
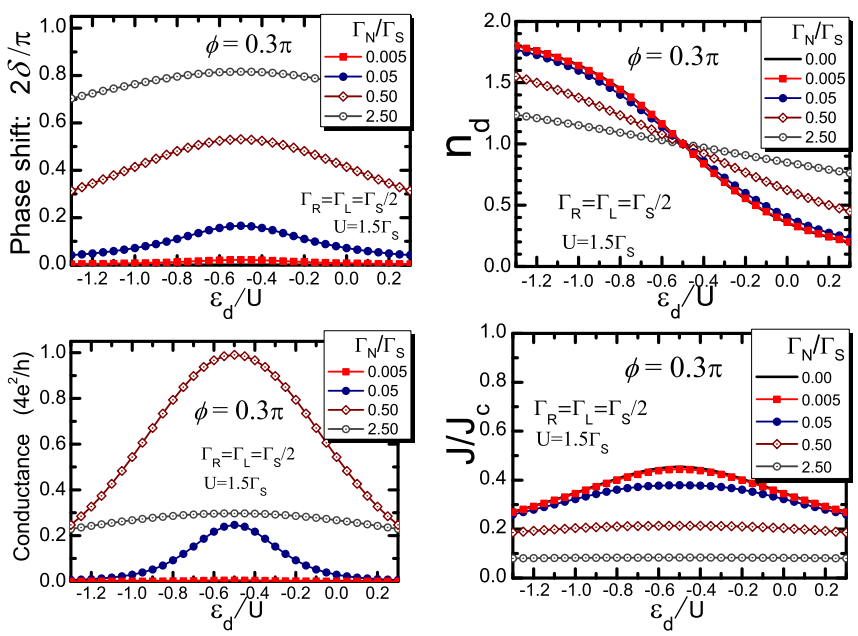

FIG. 9. (Color online) Phase shift and some related groundstate averages are plotted vs $\epsilon_{d} / U$ for $\phi=0.3 \pi$ for several values of $\Gamma_{N}$ : (upper panel) phase shift $\delta$ and electron occupation number $\left\langle n_{d}\right\rangle$, (lower panel) conductance $g_{N S}$ and Josephson current $J$ in units of $J_{C}=e \Gamma_{S} / \hbar$. The parameters are chosen to be $\Gamma_{L}=\Gamma_{R}\left(=\Gamma_{S} / 2\right)$, and $U=1.5 \Gamma_{S}$.

creases from zero to an intermediate value $\Gamma_{N} \lesssim 0.5 \Gamma_{S}$. Then, $\left\langle n_{\gamma,-1}\right\rangle$ increases as seen in the results obtained for $\Gamma_{N}=2.5 \Gamma_{S}$, and approaches 1.0 in the limit of large $\Gamma_{N}$ where this coupling dominates all the other effects. The lower right panel of Fig. 8 shows the Josephson current. This supercurrent, flowing between the two SC leads, is suppressed due to the electron correlation in the Kondo regime at $-0.82 U \lesssim \epsilon_{d} \lesssim-0.18 U$. Outside of this region the ground state is characterized by the local Cooper pairing, and the current is less suppressed although the coupling to the normal lead $\Gamma_{N}$ smears the structure due to the QPT. We also see in the lower left panel that the conductance $g_{N S}$ between the normal lead and the QD shows sharp peak at the transient region of the crossover for small $\Gamma_{N}$. The sharp conductance peak is mainly caused by the phase shift $\delta$ that changes suddenly from 0 to $\pi / 2$ at the crossover region because the conductance is proportional to $\sin ^{2} 2 \delta$. The Bogoliubov angle $\Theta_{B}$, appearing in the expression of $g_{N S}$ given in Eq. (26), varies moderately and determines the peak height.

Figure 9 shows the $\epsilon_{d}$ dependence of the correlation functions for a smaller value of the Josephson phase $\phi=0.3 \pi$. In this case $U$ is not large enough to reach the Kondo regime over the crossover region. The ground state is the local Cooper-pairing state for all values of $\epsilon_{d}$, and thus the correlation functions vary moderately as a function of the gate voltage $\epsilon_{d}$. The conductance and Josephson current have maximum at the electron-hole symmetric point $\epsilon_{d}=-0.5 U$. This is mainly because the factor $\sin \Theta_{B}$ that appears in the expression of these correlations given in Eqs. (26) and (27) takes a local maximum at the Bogoliubov angle of $\Theta_{B}=\pi / 2$.

In Fig. 10, the results of the renormalization factor $z$, the renormalized Andreev levels $\pm \widetilde{E}_{A}$, the Wilson ratio 

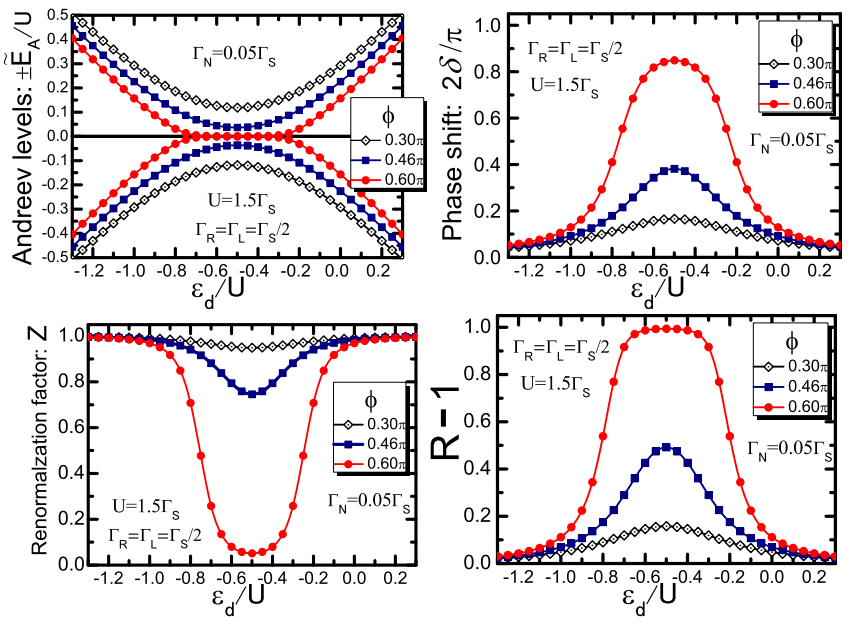

FIG. 10. (Color online) Renormalized parameters are plotted vs $\epsilon_{d} / U$ for several values of $\phi$ for relatively small coupling $\Gamma_{N}=0.05 \Gamma_{S}$ between the dot and normal lead: (upper panel) renormalized Andreev level $\pm \widetilde{E}_{A}$ and phase shift $\delta$, (lower panel) renormalization factor $z$ and Wilson ratio $R$. The other parameters are chosen such that $\Gamma_{L}=\Gamma_{R}\left(=\Gamma_{S} / 2\right)$, and $U=1.5 \Gamma_{S}$.

$R$, and the phase shift are compared for three different values for the Josephson phase $\phi=0.3 \pi, 0.46 \pi$, and $0.6 \pi$. The Coulomb interaction and the hybridization energy scales are chosen such that $U=1.5 \Gamma_{S}$ and $\Gamma_{N}=0.05 \Gamma_{S}$. We can see in the upper left panel that the pair of renormalized Andreev levels $\pm \widetilde{E}_{A}$ for $\phi=0.6 \pi$ lie very closely to the Fermi level at $-0.82 U \lesssim \epsilon_{d} \lesssim-0.18 U$. Furthermore, in this region, the renormalization factor $z$ significantly decreases and the Wilson ratio approaches $R \rightarrow 2$ owing to the strong correlations in the Kondo regime. It also indicates that the Kondo temperature $T_{K}=\pi \widetilde{\Gamma}_{N} / 4$ and the renormalized resonance width $\widetilde{\Gamma}_{N}$, defined in Eq. (18), become very small. Simultaneously, the local Bogoliubov particle number on the dot approaches single occupancy $\left\langle n_{\gamma,-1}\right\rangle=2 \delta / \pi \simeq 0.9$ although it is less than 1.0 because $\Gamma_{N}$ is not very small in this case. Note that the sharp single Kondo peak that we have seen in Fig. 5 for $U \gtrsim 15 \Gamma_{N}$ consists of such a pair of renormalized Andreev levels, appearing in the close vicinity of the Fermi level.

The ground-state properties show a marginal behavior at $\phi=0.46 \pi$, as shown in Fig. 10. For instance, $z$ and $R$ take the intermediate values, and the pair of $\pm \widetilde{E}_{A}$ becomes distinguishable as we can see in the upper left panel of Fig. 10. Then, for a smaller value of the Josephson phase $\phi=0.3 \pi$, the ground state is a singlet caused by the local Cooper pairing, as mentioned. Therefore, in this case the electron correlations are suppressed as $z \simeq 0.95$ and $R \simeq 1.16$ even at the electron-hole symmetric point $\epsilon_{d}=-0.5 U$.
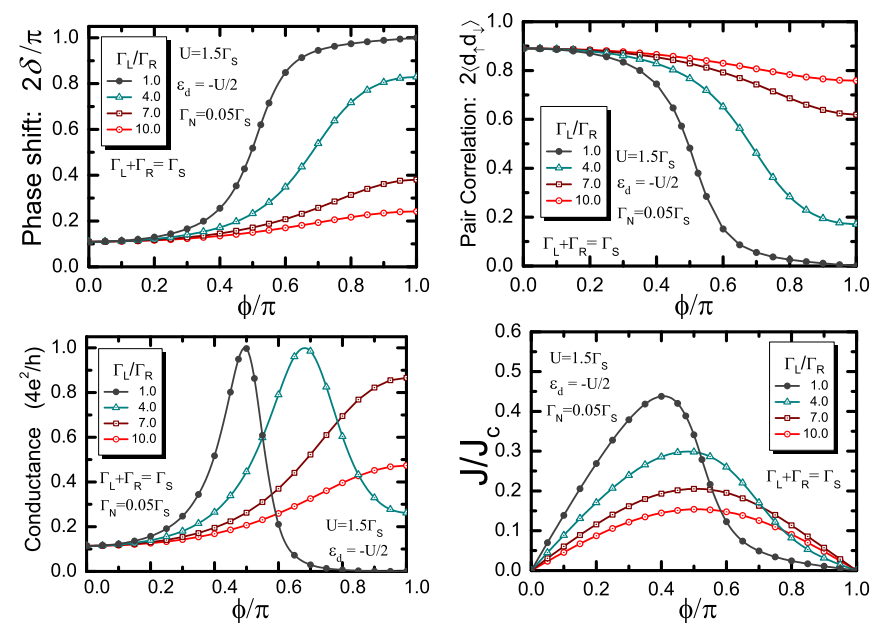

FIG. 11. (Color online) Phase shift and some related ground-state averages for the asymmetric couplings $\Gamma_{R} \neq \Gamma_{L}$ are plotted vs Josephson phase $\phi$ for several $\Gamma_{L} / \Gamma_{R}$ keeping $\Gamma_{S}=\Gamma_{R}+\Gamma_{L}$ unchanged: (upper panel) phase shift $\delta$ and pair correlation $2\left|\left\langle d_{\downarrow} d_{\uparrow}\right\rangle\right|$, (lower panel) conductance $g_{N S}$ and (lower right) Josephson current in units of $J_{C}=e \Gamma_{S} / \hbar$. The other parameters are chosen such that $\epsilon_{d}=-U / 2, U=1.5 \Gamma_{S}$ and $\Gamma_{N}=0.05 \Gamma_{S}$.

\section{Spatial asymmetry in the junction $\left(\Gamma_{L} \neq \Gamma_{R}\right)$}

So far, we have assumed that the Josephson junction is symmetric $\Gamma_{L}=\Gamma_{R}$. However, the SC proximity effects in real systems depend on the asymmetry in the couplings $\Gamma_{L} \neq \Gamma_{R}$. One difference of the asymmetric junction from the symmetric one is that the maximum possible of the transmission probability is no longer one, namely $\mathcal{T}_{0}<1$. Specifically, the local SC gap induced on the dot $\left|\Delta_{d}\right|$, defined in Eq. (7), becomes larger in the asymmetric junction than that in the symmetric junction for which $\mathcal{T}_{0}=1$. Therefore, the asymmetry in the couplings tends to enhance the SC proximity effects as it suppresses the reduction of $\left|\Delta_{d}\right|$ due to the Josephson phase $\phi$. In the following, we discuss the ground state properties of the asymmetric junction with $\Gamma_{L} \neq \Gamma_{R}$, keeping the sum $\Gamma_{S}=\Gamma_{R}+\Gamma_{L}$ unchanged at $\Gamma_{N}=0.05 \Gamma_{S}$. For simplicity, we examine the electron-hole symmetric case $\epsilon_{d}=-U / 2$, where the bare Andreev level is given by $E_{A}=\left|\Delta_{d}\right|$ and the Bogoliubov angle is fixed at $\Theta_{B}=\pi / 2$.

The NRG results for the asymmetric junction are plotted vs the Josephson phase $\phi$ in Fig. 11 for $U=1.5 \Gamma_{S}$. In each panel, the curve for $\Gamma_{L} / \Gamma_{R}=1.0$ corresponds to the results obtained for the symmetric coupling, presented also in Fig. 6. As mentioned in the above, the amplitude of the static SC gap $\left|\Delta_{d}\right|$ for the asymmetric junction becomes larger that for the symmetric junction. Thus the position of $E_{A}$ moves away from the Fermi energy due to the spatial asymmetry, and this causes rather moderate $\phi$ dependence of the phase shift $\delta$ for $\Gamma_{L} \neq \Gamma_{R}$, seen in the upper left panel of Fig. 11. Correspondingly, the SC pair correlation $2\left\langle d_{\uparrow} d_{\downarrow}\right\rangle$, shown in the upper right 
panel, increases as the asymmetry $\Gamma_{L} / \Gamma_{R}$ increases.

The conductance due to the Andreev scattering is proportional to $\sin ^{2} 2 \delta$, and thus has a peak when the phase shift takes the value of $\delta=\pi / 4$ at crossover region between the local-Cooper pairing and Kondo singlet states. We see in the lower left panel of Fig. 11 that the position of the conductance peak shifts towards the larger $\phi$ side as $\delta$ becomes smaller with increasing $\Gamma_{L} / \Gamma_{R}$. This also shows that the asymmetric coupling favors the SC proximity into the dot, and enlarges the parameter region for the local-Cooper-pairing ground state. Furthermore, the crossover behavior from the local-Cooper pairing to the Kondo regime, seen for the Josephson current in the lower right panel for $\Gamma_{L} / \Gamma_{R}=1$, is smeared as $\Gamma_{L} / \Gamma_{R}$ increases, and the current shows a simple sinusoidal $\phi$ dependence for large asymmetries.

\section{SUMMARY}

We have studied the crossover between a Kondo singlet and a local-Copper-pairing singlet, occurring in a quantum dot coupled to one normal and two SC leads. The low-energy states of the system can be described by excitations from a local Fermi-liquid ground state of interacting Bogoliubov particles. Specifically in this three terminal configuration, the renormalized parameters for the quasiparticles vary as functions of the Josephson phase $\phi$, and the crossover occurs at finite $\phi_{C}$ as the phase difference varies in the range $0 \leq|\phi| \leq \pi$. We have calculated the phase shift $\delta$, the renormalization factor $z$, the renormalized Andreev level $\pm \widetilde{E}_{A}$, and the Wilson raton $R$ in the large SC gap limit $\Delta_{\mathrm{SC}} \rightarrow \infty$, using the NRG, and have deduced the transport properties at $T=0$.

The Bogoliubov particles are strongly renormalized in the Kondo regime while the renormalization is a minor effect in the local-Cooper-pairing regime which corresponds to the frozen-impurity fixed point of the NRG. Near the crossover between the two regimes a pair of renormalized Andreev levels $\pm \widetilde{E}_{A}$ approach the Fermi level, and the conductance between the dot and normal lead has a peak. The Josephson current between the two SC leads is suppressed significantly in the Kondo regime.

We have also presented the spectral function calculated with the NRG. The results demonstrate precise features of the original Andreev levels, which for the local-Cooperpairing state are broadened by $\Gamma_{N}$ and then renormalized as $U$ increases. In the Kondo regime, the pair of renormalized Andreev levels overlap to form a single peak near the Fermi level while at high energies four additional peaks are visible. These peaks correspond to the excitations to the upper and lower atomic peaks, which are defined with respect to the Bogoliubov particles and consist of a linear combination of the empty and doubly occupied electron states. Thus, the broadened bare Andreev peaks and a low energy feature corresponding to a Kondo resonance can appear within the superconducting gap. For suitable parameters this should be observable experimentally in the discussed Y-shape geometry.

\section{ACKNOWLEDGMENTS}

We thank Yasuhiro Yamada, Rui Sakano, R. S. Deacon, A. C. Hewson, and N. Kawakami for valuable discussions. A.O. is supported by JSPS Grant-in-Aid for Scientific Research C (No. 23540375). Y.T. was supported by Special Postdoctoral Researchers Program of RIKEN. J.B. acknowledges financial support from the DFG through the grant BA 4371/1-1. Numerical computation was partly carried out at Yukawa Institute Computer Facility.

\section{Appendix A: Current in the large gap limit}

We provide the expression of the Josephson current in the large gap limit in this appendix. To this end, we use the imaginary time Green's function, defined by

$$
\boldsymbol{G}_{d d}(\tau)=-\left[\begin{array}{ll}
\left\langle T_{\tau} d_{\uparrow}(\tau) d_{\uparrow}^{\dagger}\right\rangle & \left\langle T_{\tau} d_{\uparrow}(\tau) d_{\downarrow}\right\rangle \\
\left\langle T_{\tau} d_{\downarrow}^{\dagger}(\tau) d_{\uparrow}^{\dagger}\right\rangle & \left\langle T_{\tau} d_{\downarrow}^{\dagger}(\tau) d_{\downarrow}\right\rangle
\end{array}\right] .
$$

The Fourier transform of this function can be expressed in the form,

$$
\begin{aligned}
\left\{\boldsymbol{G}_{d d}(i \omega)\right\}^{-1}= & i \omega \mathbf{1}-\xi_{d} \boldsymbol{\tau}_{3}+i \Gamma_{N} \operatorname{sgn} \omega \mathbf{1} \\
& -\sum_{\nu=L, R} v_{\nu}^{2} \boldsymbol{g}_{\nu}(i \omega)-\boldsymbol{\Sigma}(i \omega)
\end{aligned}
$$

Here, $i \omega$ is the Matsubara frequency, $\boldsymbol{\Sigma}(i \omega)$ is the self energy due to the Coulomb interaction, $\boldsymbol{g}_{\nu}(i \omega)$ is the local Green's function at the junction of the lead $\nu$

$$
\boldsymbol{g}_{\nu}(i \omega)=-\pi \rho_{\nu} \frac{i \omega \mathbf{1}-\boldsymbol{\Delta}_{\nu}}{\sqrt{\omega^{2}+\left|\Delta_{\nu}\right|^{2}},} \quad \boldsymbol{\Delta}_{\nu} \equiv\left[\begin{array}{cc}
0 & \Delta_{\nu} \\
\Delta_{\nu}^{*} & 0
\end{array}\right]
$$

1 and $\tau_{3}$ are the unit and Pauli matrices, respectively.

The Josephson current from the dot to the SC lead for $\nu=L, R$ can be expressed in terms of the Green's function

$$
\begin{aligned}
\left\langle J_{\nu}\right\rangle= & \frac{e}{\hbar} \frac{i v_{\nu}^{2}}{\beta} \\
& \times \sum_{\omega_{n}} \operatorname{Tr}\left[\left\{\boldsymbol{g}_{\nu}\left(i \omega_{n}\right) \boldsymbol{\tau}_{3}-\boldsymbol{\tau}_{3} \boldsymbol{g}_{\nu}\left(i \omega_{n}\right)\right\} \boldsymbol{G}_{d d}\left(i \omega_{n}\right)\right] .
\end{aligned}
$$

In the limit of $\left|\Delta_{\nu}\right| \rightarrow \infty$, the lead Green's function becomes a constant $\boldsymbol{g}_{\nu} \rightarrow \pi \rho_{\nu} \boldsymbol{\Delta}_{\nu} /\left|\Delta_{\nu}\right|$ as the retardation effects caused by the $\omega$ dependence in Eq. (A3) are suppressed. Then, Eq. (A4) can be rewritten in the form,

$$
\begin{aligned}
\left\langle J_{\nu}\right\rangle & \rightarrow \frac{e}{\hbar} 4 \Gamma_{\nu} \frac{e^{i \theta_{\nu}}\left\langle d_{\downarrow} d_{\uparrow}\right\rangle-e^{-i \theta_{\nu}}\left\langle d_{\uparrow}^{\dagger} d_{\downarrow}^{\dagger}\right\rangle}{2 i} \\
& =\frac{e}{\hbar} 4 \Gamma_{\nu}\left|\left\langle d_{\downarrow} d_{\uparrow}\right\rangle\right| \sin \left(\theta_{\nu}-\theta_{d}\right) .
\end{aligned}
$$


Note that $\left\langle d_{\downarrow} d_{\uparrow}\right\rangle=\left|\left\langle d_{\downarrow} d_{\uparrow}\right\rangle\right| e^{i \theta_{d}}$, in the limit of large gap as the phase of $\left\langle d_{\downarrow} d_{\uparrow}\right\rangle$ coincides with that of the local SC gap $\Delta_{d}=\left|\Delta_{d}\right| e^{i \theta_{d}}$ as shown in Eq. (25). The current conservation $\left\langle J_{R}\right\rangle+\left\langle J_{L}\right\rangle=0$ can be confirmed explicitly through the identity

$$
\Gamma_{R} \sin \left(\theta_{R}-\theta_{d}\right)-\Gamma_{L} \sin \left(\theta_{d}-\theta_{L}\right)=0,
$$

which follows from the definition of $\theta_{d}$ given in Eq. (6).
Using Eq. A7 , the current can be expressed in the form

$$
\langle J\rangle=\frac{e}{\hbar} 4 \Gamma_{R} \Gamma_{L} \frac{\left|\left\langle d_{\downarrow} d_{\uparrow}\right\rangle\right|}{\left|\Delta_{d}\right|} \sin \phi .
$$

This can be rewritten further, in terms of the phase shift $\delta$ and the Bogoliubov angle $\Theta_{B}$, as shown in Eq. (27).
${ }^{1}$ H. Shiba and T. Soda, Prog. Theor. Phys. 41, 25 (1969).

${ }^{2}$ E. Müller-Hartmann and J. Zittartz, Z. Phys. 234, 58 (1970).

3 T. Matsuura, Prog. Theor. Phys. 57, 1823 (1977).

4 M. Jarrell, D. S. Sivia and B Patton, Phys. Rev. B 42, 4804 (1990).

${ }^{5}$ K. Satori, H. Shiba, O. Sakai, and Y. Shimizu, J. Phys. Soc. Jpn. 61, 3239 (1992)

${ }^{6}$ T. Yoshioka and Y. Ohashi, J. Phys. Soc. Jpn. 69, 1812 (2000).

7 J. Bauer, A. Oguri, and A. C. Hewson, J. Phys.: Condes. Mat. 19, 486211 (2007).

8 T. Meng, S. Florens, and P. Simon, Phys. Rev. B 79224521 (2009).

9 J. Bauer, J. I. Pascual, and K. J. Franke, arXiv:1208.3211

10 A. Martín-Rodero and A. Levy Yeyati, Adv. Phys. 60, 899 (2011).

11 M. R. Buitelaar, T. Nussbaumer and C. Schönenberger, Phys. Rev. Lett. 89, 256801 (2002).

12 A. Eichler, M. Weiss, S. Oberholzer, C. Schönenberger, A. Levy Yeyati, J. C. Cuevas, and A. Martín-Rodero, Phys. Rev. Lett. 99, 126602 (2007).

13 T. Sand-Jespersen, J. Paaske, B. M. Andersen, K. GroveRasmussen, H. I. Jørgensen, M. Aagesen, C. B. Sørensen, P. E. Lindelof, K. Flensberg, and J. Nygård, Phys. Rev. Lett. 99, 126603 (2007).

14 C. Buizert, A. Oiwa, K. Shibata, K. Hirakawa, and S. Tarucha, Phys. Rev. Lett. 99, 136806 (2007).

15 K. Grove-Rasmussen, H. I. Jørgensen, and P. E. Lindelof, New J. Phys. 9, 124 (2007).

16 S. Ishizaka, J. Sone, and T. Ando, Phys. Rev. B 52, 8358 (1995).

17 A. A. Clerk and V. Ambegaokar, Phys. Rev. B 61, 9109 (2000)

18 A. V. Rozhkov and D. P. Arovas, Phys. Rev. Lett. 82, 2788 (1999)

19 Y. Avishai, A. Golub and A. D. Zaikin, Phys. Rev. B 67, 041301 (2003).

20 E. Vecino, A. Martin-Rodero, and A. Levy Yeyati, Phys. Rev. B 68, 035105 (2003).

21 K. Kusakabe, Y. Tanaka, and Y. Tanuma, Physica E, 18, 50 (2003).

${ }^{22}$ F. Siano and R. Egger, Phys. Rev. Lett. 93, 047002 (2004).

23 M-S. Choi, M. Lee, K. Kang, and W. Belzig, Phys. Rev. B 70, 020502 (2004).

24 A. Oguri, Yoshihide Tanaka and A. C. Hewson, J. Phys. Soc. Jpn. 73, 2494 (2004).

25 Yoshihide Tanaka, A. Oguri, and A. C. Hewson, New J. Phys. 9, 115 (2007); 10, 029801(E) (2008).

${ }^{26}$ C. Karrasch, A. Oguri, and V. Meden, Phys. Rev. B 77,
024517 (2008).

27 T. Hecht, A. Weichselbaum, J. von Delft, R. Bulla, J. Phys.: Condes. 20, 275213 (2008).

28 D. J. Luitz, F. F. Assaad, T. Novotný, C. Karrasch, and V. Meden, Phys. Rev. Lett. 108, 227001 (2012).

29 M. R. Gräber, T. Nussbaumer, W. Belzig, and C. Schönenberger, Nanotechnology 15, S479 (2004).

30 R. S. Deacon, Yoichi Tanaka, A. Oiwa, R. Sakano, K. Yoshida, K. Shibata, K. Hirakawa, and S. Tarucha, Phys. Rev. B 81, 121308 (2010).

31 R. Fazio and R. Raimondi, Phys. Rev. Lett. 80, 2913 (1998); 82, 4950(E) (1999).

32 P. Schwab and R. Raimondi, Phys. Rev. B 59, 1637 (1999).

33 S. Y. Cho, K. Kang, and C.-M. Ryu, Phys. Rev. B 60, 16874 (1999).

34 A. A. Clerk, V. Ambegaokar, and S. Hershfield, Phys. Rev. B 61, 3555 (2000).

35 Q.-F. Sun, H. Guo, and T.-H. Lin, Phys. Rev. Lett. 87, 176601 (2001).

36 J. C. Cuevas, A. Levy Yeyati, and A. Martín-Rodero, Phys. Rev. B 63, 094515 (2001).

37 Y. Avishai, A. Golub, and A. D. Zaikin Phys. Rev. B 63, 134515 (2001); Europhys. Lett. 55, 397 (2001).

38 T. Aono, A. Golub, and Y. Avishai, Phys. Rev. B 68, 045312 (2003).

39 M. Krawiec and K. I. Wysokiński, Supercond. Sci. Technol. 17, 103 (2004).

40 J. Splettstoesser, M. Governale, J. König, F. Taddei, and R. Fazio, Phys. Rev. B 75, 235302 (2007).

41 T. Domański, A. Donabidowicz, and K.I. Wysokiński, Phys. Rev. B 76, 104514 (2007); 78, 144515 (2008); T. Domański, A. Donabidowicz, ibid. 78, 073105 (2008).

42 Yoichi Tanaka, N. Kawakami, and A. Oguri, J. Phys. Soc. Jpn. 76, 074701 (2007); 77, 098001(E) (2008).

43 Yoichi Tanaka, N. Kawakami, and A. Oguri, Phys. Rev. B 78, 035444 (2008).

44 Yoichi Tanaka, N. Kawakami, and A. Oguri, Phys. Rev. B 81, 075404 (2010).

45 L. Hofstetter, S. Csonka, J. Nygård, and C. Schönenberger, Nature 461, 960 (2009).

46 L. G. Herrmann, F. Portier, P. Roche, A. Levy Yeyati, T. Kontos, and C. Strunk, Phys. Rev. Lett. 104, 026801 (2010)

47 W. Chang, V. E. Manucharyan, T. S. Jespersen, J. Nygård, and C. M. Marcus, arXiv:1211.3954

48 M. G. Pala, M. Governale and J. König, New. J. Phys. 9, 278 (2007).

49 M. Governale, M. G. Pala, and J. König, Phys. Rev. B 77, 134513 (2008)

50 A. Oguri and Yoichi Tanaka, J. Phys. Conference Series 
391, 012146 (2012).

${ }^{51}$ H. R. Krishna-murthy, J. W. Wilkins, and K. G. Wilson, Phys. Rev. B 211044 (1980).

52 A. C. Hewson, The Kondo Problem to Heavy Fermions (Cambridge University Press, Cambridge, 1993).

53 M. R. Galpin, and D. E. Logan, Phys. Rev. B 77, 195108 (2008).
54 I. Affleck, J. -S. Caux, and A. M. Zagoskin, Phys. Rev. B 62, $1433(2000)$.

55 J. S. Langer and V. Ambegaokar, Phys. Rev. 121, 1090 (1961).

56 H. Shiba, Prog. Theor. Phys. 54, 967 (1975).

57 A. C. Hewson, A. Oguri, and D. Meyer, Eur. Phys. J. B 40, 177 (2004). 